\title{
Re-Evaluation of the Safety of Laser-Assisted Subepithelial Keratectomy
}

\author{
Yuji Kumano1, Yumi Soejima', Shinichiro Numa1, Hiroyasu Matsui', Ikuko Zushi², \\ Masahito Shigematsu' ${ }^{3}$, Takaaki Matsui' ${ }^{1}$, Teruo Nishida1 \\ ${ }^{1}$ Ohshima Hospital of Ophthalmology, Fukuoka, Japan \\ ${ }^{2}$ Zushi Eye Clinic, Fukuoka, Japan \\ ${ }^{3}$ Department of Oral and Maxillofacial Surgery, Saga University, Saga, Japan \\ Email:yujik@car.ocn.ne.jp
}

Received 18 May 2014; revised 28 June 2014; accepted 24 July 2014

Copyright (C) 2014 by authors and Scientific Research Publishing Inc.

This work is licensed under the Creative Commons Attribution International License (CC BY).

http://creativecommons.org/licenses/by/4.0/

(c) (i) Open Access

\begin{abstract}
Purpose: Additional analyses of outcomes of laser-assisted subepithelial keratectomy (LASEK) are still necessary to improve the safety of LASEK. Therefore, in our study, outcomes were assessed retrospectively in 561 eyes that underwent LASEK treatment. Methods: Uncorrected visual acuity (UCVA), best spectacle-corrected visual acuity (BSCVA) and residual spherical equivalent were analyzed at 3 and 6 months postoperatively. We assessed four subgroups based on the degree of preoperative myopia considering mean BSCVA and loss of two or more lines. Results: Mean UCVAs and BSCVAs were obtained at 3 and 6 months postoperatively $(1.23 / 1.35$ and $1.23 / 1.37$, respectively). The mean predictability was within \pm 0.125 diopters. Conversely, the safety indexes were 0.94 and 0.96 respectively, and the efficacy indexes were 0.86 and 0.86 at 3 and 6 months postoperatively, respectively. 8.4\% eyes and 5.2\% eyes lost two or more lines of BSCVA at 3 and 6 months postoperatively, respectively. The mean BSCVAs of the high or ultra-high groups were significantly lower than those of the low or mild groups both 3 months and 6 months postoperatively. The incidence rates of losing two or more lines of BSCVA in the high or ultra-high myopia groups were significantly greater than in the low or mild groups at 3 months and 6 months postoperatively. Conclusion: LASEK predictably corrected myopia achieving $>1.2$ in UCVA and BSCVA. However, the safety and efficacy indexes were $<1.0$, which were related to the loss of two or more lines of BSCVA. Since the incidence rates of losing two or more lines of BSCVA were greater in the high or ultra-high myopia groups, we consider LASEK as more safely performed in patients with low to mild preoperative myopia.
\end{abstract}

\section{Keywords}

BSCVA, LASEK, Loss of Two or More Lines, Preoperative Myopia, Re-Evaluation

How to cite this paper: Kumano, Y., Soejima, Y., Numa, S., Matsui, H., Zushi, I., Shigematsu, M., Matsui, T. and Nishida, T. (2014) Re-Evaluation of the Safety of Laser-Assisted Subepithelial Keratectomy. Open Journal of Ophthalmology, 4, 79-85. 


\section{Introduction}

Laser-assisted subepithelial keratectomy (LASEK) combines the advantages of photorefractive keratectomy (PRK) and laser-assisted in situ keratomileusis (LASIK) while avoiding the disadvantages of LASIK. It has no flap-related complications, reduces the risk of keratectasia associated with LASIK and had been shown to be a safe, effective and predictable treatment for low [1], mild [2] and high [3]-[6] myopia from its introduction [7] [8]. Nevertheless, a clinically significant haze has occurred in $>30 \%$ [7] [9] of eyes at 3 months, 6.9\% [4] of at 6 months and 3.8\% [10] of eyes at 1 year. Moreover, $14.3 \%$ of eyes reportedly lose more than one line after LASEK compared with $1.2 \%$ for LASIK at 1 year [6]. The efficacy of mitomycin C (MMC) [11] in reducing the incidence of these complications has led to its widespread use in most refractive surgery practices. However, the mechanism of action and possible toxicity of this drug remain subjects of intense debate. Therefore, there is controversy about the outcomes of LASEK [12]-[14].

We believe additional analysis of outcomes of LASEK without MMC is still necessary to improve the safety of LASEK. Therefore, LASEK outcomes were assessed retrospectively and the mean best spectacle-corrected visual acuity (BSCVA) and loss of two or more lines following LASEK were evaluated according to the degree of preoperative myopia.

\section{Materials and Methods}

\subsection{Patients}

The originally considered 821 eyes underwent LASEK between November 2000 and November 2011. All eyes with a history of previous refractive surgery, preoperative BSCVA of 0.9 or worse, and that had undergone the procedure with the aim of near correction were excluded. Eyes in which decimal visual acuities were not measured at both 3 months and 6 months postoperatively were also excluded. Two hundred and sixty eyes were excluded and 561 eyes remained in the study and were analyzed. The mean patient age was $30.2 \pm 8.5$ years (range 16 - 61 years). Three hundred and eighteen patients (56.7\%) were male and 243 (43.3\%) were female.

\subsection{Surgical Procedure}

A standard LASEK technique, as described previously, was used [7]. In brief, after topical anesthesia, positioning marks were made on the corneal surface and a circular well was used to administer $20 \%$ alcohol for 20 - 30 seconds to the corneal epithelial surface. The loosened epithelium was then peeled back using a spatula. All photorefractive ablations were performed with the VISX Star Excimer Laser System (AMO, Abbott Park, IL, USA). After standard laser, the epithelial sheet was gently repositioned and the epithelium was carefully realigned and allowed to dry. A combination of antibiotics and steroid eye drops was then administered, followed by insertion of a bandage contact lens to reduce mechanical friction by the eyelid and postoperative pain. The postoperative regimen included topical antibiotics, diclofenac sodium and topical steroid eye drops four times per day in all patients and short-term systemic steroids in some patients to reduce the initial inflammatory damage to the corneal stroma. The topical eye drops were continued for 3 months after surgery and then were tapered off.

\subsection{Analysis of Data}

The LASEK outcomes were assessed at 3 months and 6 months postoperatively. The safety index of LASEK is defined as mean postoperative BSCVA/mean preoperative BSCVA. The efficacy index is defined as mean postoperative uncorrected visual acuity (UCVA)/mean preoperative BSCVA. Predictability is evaluated by postoperative mean spherical equivalents (SE). Mean BSCVA and loss of two or more lines of BSCVA were also compared between four subgroups composed of various degrees of preoperative SE: low [-3.00 diopters (D) or less], mild [from $-3.125 \mathrm{D}$ to $-6.00 \mathrm{D}$ ], high [from $-6.125 \mathrm{D}$ to $-10.00 \mathrm{D}$ ], and ultra-high [more than $-10.125 \mathrm{D}]$. Visual acuity was converted to the logarithm of the minimum angle of resolution (logMAR) from the decimal notation for statistical analysis. Continuous data are expressed as the mean \pm standard deviation. Statistical comparisons were done with the multiple comparison procedure by Bonferroni adjustment or with Fisher's exact test. $\mathrm{P} \leq 0.05$ was considered statistically significant.

\section{Results}

Preoperative data are shown in Table 1 and postoperative data are shown in Table 2. The mean decimal visual 
Table 1. Preoperative findings in 561 eyes (BSCVA: best spectacle-corrected visual acuity; D: diopters; SD: standard devia-

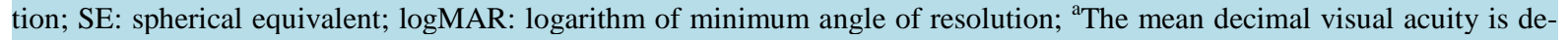
rived from the mean logMAR).

\begin{tabular}{ccc}
\hline & Parameter & Outcome \\
\hline Refractive error & Sphere range (D) & 1.25 to -14.25 \\
Mean sphere \pm SD (D) & $-5.77 \pm 2.71$ \\
Cylinder range (D) & 0.00 to -6.50 \\
Mean cylinder \pm SD (D) & $-0.88 \pm 0.84$ \\
SE range (D) & -1.00 to -14.25 \\
Mean SE \pm SD (D) & $-6.21 \pm 2.73$ \\
BSCVA & Range & 1.0 to 2.0 \\
& Mean logMAR BSCVA \pm SD & $-0.157 \pm 0.045$ \\
& a Mean decimal visual acuity & 1.43 \\
\hline
\end{tabular}

Table 2. Postoperative refractive and visual outcomes of 561 eyes (UCVA: uncorrected visual acuity; BSCVA: best spectacle-corrected visual acuity; SE: spherical equivalent; logMAR: logarithm of minimum angle of resolution; SD: standard

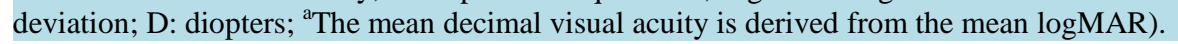

\begin{tabular}{cccc}
\hline & & \multicolumn{2}{c}{ Outcome } \\
\hline & Parameter & 3 months & 6 months \\
\hline UCVA & Range & 0.2 to 2.0 & 0.1 to 2.0 \\
& UCVA of 1.0 or better (\% of eyes) & 491 eyes (87.5\%) & 490 eyes (87.3\%) \\
& Mean logMAR UCVA \pm SD & $-0.090 \pm 0.118$ & $-0.089 \pm 0.136$ \\
BSCVA & ${ }^{a}$ Mean decimal visual acuity & 1.23 & 1.23 \\
& Range & 0.5 to 2.0 & 0.3 to 2.0 \\
BSCVA of 1.0 or better (\% of eyes) & 540 eyes (96.2\%) & 555 eyes (98.9\%) \\
Residual SE & Mean logMAR BSCVA \pm SD & $-0.131 \pm 0.073$ & $-0.137 \pm 0.070$ \\
a Mean decimal visual acuity & Range (D) & 1.35 & 1.37 \\
Efficacy index & Mean SE \pm SD (D) & +2.25 to -2.00 & +1.25 to -4.125 \\
\hline
\end{tabular}

acuity (VA) of postoperative UCVAs were 1.23 and the mean decimal VA of postoperative BSCVAs were $>1.35$ at 3 months and 6 months postoperatively. The mean predictabilities were within $\pm 0.125 \mathrm{D}$ at 3 months and 6 months postoperatively. Conversely, the safety indexes were 0.94 and 0.96 , and the efficacy indexes were 0.86 and 0.86 at 3 months and 6 months postoperatively, respectively. On closer scrutiny of the safety, we found that 47/561 (8.4\%) and 29/561 eyes (5.2\%) lost two or more lines of BSCVA at 3 months and 6 months postoperatively, respectively. Twenty-four of 47 (51.1\%) and 19 of 29 (65.5\%) eyes of those that lost two or more lines of BSCVA at 3 months and 6 months postoperatively showed various grades of corneal haze.

To compare visual outcomes according to degree of myopia, the eyes were classified into four subgroups based on degree of preoperative SE: low $(n=74)$, mild $(n=216)$, high $(n=218)$ and ultra-high $(n=53)$. The mean logMARs of BSCVAs (converted to decimal VA) were $-0.155 \pm 0.052$ (1.43), $-0.153 \pm 0.060$ (1.42), $-0.111 \pm 0.078(1.29)$, and $-0.084 \pm 0.082(1.21)$ at 3 months postoperatively, and $-0.161 \pm 0.044(1.45)$, $-0.162 \pm 0.049(1.45),-0.117 \pm 0.069(1.31)$, and $-0.086 \pm 0.107$ (1.22) at 6 months postoperatively in the low, mild, high and ultra-high myopia groups, respectively. Each decimal VA when converted from mean logMAR 
of the BSCVA was between 1.2 and 1.5 (Figure 1). The mean BSCVAs of the high or ultra-high groups were significantly lower than those of the low or mild groups both 3 months and 6 months postoperatively $(\mathrm{p}<0.01$ with the multiple comparison procedure by Bonferroni adjustment). Losing two or more lines of BSCVA occurred in 2/74 (2.7\%), 9/216 (4.2\%), 25/218 (11.5\%), and 11/53 (20.8\%) eyes at 3 months postoperatively and in 0/74 (0\%), 2/216 (0.9\%), 22/218 (10.1\%), and 5/53 (9.4\%) eyes at 6 months postoperatively in the low, mild, high and ultra-high myopia groups, respectively (Figure 2). The rates of losing two or more lines of BSCVA in the high or ultra-high groups at 3 months postoperatively were significantly higher than those of the low or mild groups 3 months postoperatively ( $<0.05$ with Fisher's exact test). The rates of losing two or more lines of BSCVA in the high or ultra-high groups were significantly higher than those of the low or mild groups

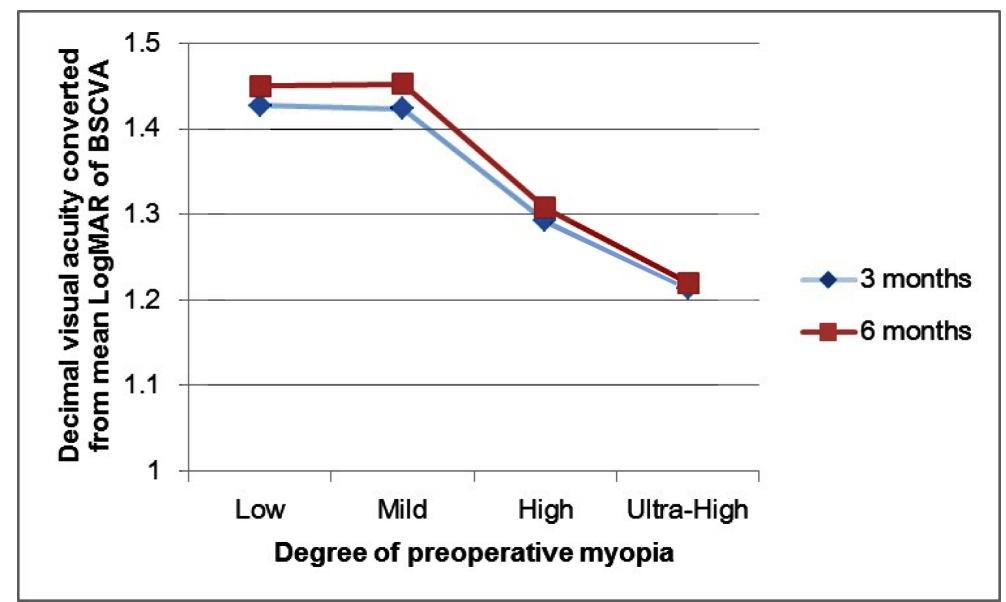

Figure 1. Decimal visual acuity converted from mean logMAR BSCVA at 3 months and 6 months postoperatively according to the degree of preoperative spherical equivalent. The mean BSCVAs of the high or ultra-high groups are significantly ( $p<0.01$ with the multiple comparison procedure by Bonferroni adjustment) lower than those of the low or mild groups both 3 months and 6 months postoperatively. Low: -3.00 diopters (D) or less $(n=74)$; mild: $-3.125 \mathrm{D}$ to $-6.00 \mathrm{D}(\mathrm{n}=216)$; high: $-6.125 \mathrm{D}$ to $10.00 \mathrm{D}(\mathrm{n}=220)$; ultra-high: more than $-10.125 \mathrm{D}(\mathrm{n}=53)$; BSCVA: best spectacle-corrected visual acuity; logMAR: logarithm of minimum angle of resolution.

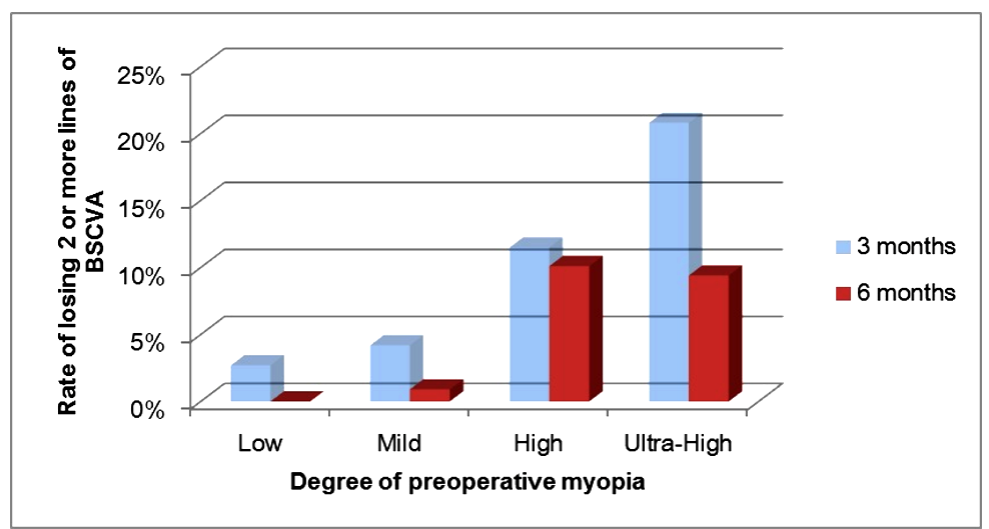

Figure 2. Rate of loss of two or more lines of BSCVA at 3 months and 6 months postoperatively according to the degree of preoperative spherical equivalent. The rates in the high or ultra-high groups are significantly higher than those in the other groups ( $p<0.05$ with Fisher's exact test) both 3 months and 6 months postoperatively. Low: -3.00 diopters (D) or less ( $\mathrm{n}=$ 74); mild: $-3.125 \mathrm{D}$ to $-6.00 \mathrm{D}(\mathrm{n}=216)$; high: $-6.125 \mathrm{D}$ to $10.00 \mathrm{D}(\mathrm{n}=$ 220); ultra-high: more than $-10.125 \mathrm{D}(\mathrm{n}=53)$; BSCVA: best spectaclecorrected visual acuity. 
at 6 months postoperatively ( $\mathrm{p}<0.05$ with Fisher’s exact test).

\section{Discussion}

Recently, analyses of large numbers of patients treated with LASEK have been published [7] [15] [16]. Zhao et al. [15] reported a comprehensive literature search using Cochrane Collaboration methodology to identify randomized controlled trials of PRK (499 eyes) versus LASEK (512 eyes); however, this study had the drawbacks of being a systematic review and meta-analysis. Bentio-Llopis et al. [16] evaluated 1036 eyes treated with LASEK in a single institution and their data showed LASEK is definitely effective, safe and predictably corrects myopia. However, because they used MMC $0.02 \%$ in 80.7\% of 1036 eyes treated with LASEK, which would have modified the outcomes, it is not possible to conclude whether LASEK without MMC is effective or safe. In our study, we were able to retrospectively evaluate the outcomes of LASEK (561 eyes) without administering MMC in a single institution where surgical nomogram, laser systems and intraoperative and postoperative procedures were identical, thus avoiding many potentially confounding factors. Consequently the safety index and efficacy index were $<1.0$, although the mean decimal VA of postoperative UCVA was 1.23 and the mean decimal VA of postoperative BSCVA was $>1.35$. We found losing two or more lines of BSCVA occurred as well. Therefore we discuss how to perform LASEK in a safer manner.

In regard to losing two or more lines of BSCVA, Taneri et al. [7] reported that only one eye of 1421 eyes $(<0.1 \%)$ lost two lines of BSCVA. This rate is very low compared with the rate reported by Bentio-Llopis et al. [16] or our results. One possible explanation for this discrepancy is that Taneri et al. derived their data from a literature review. A larger series than reported here is needed to detect any statistically significant adverse outcomes of visual loss. The report of Bentio-Llopis et al. [16] is very important in that, in their study, 23 of 1036 eyes $(1.93 \%)$ and 10 eyes of 1036 eyes (0.96\%) lost two or more lines of BSCVA at 3 months and 6 months postoperatively, respectively. This is low compared with our findings that 47/561 (8.4\%) and 29/561 eyes (5.2\%) lost two or more lines of BSCVA at 3 months and 6 months postoperatively, respectively. One possible explanation for this discrepancy is the different frequency of use of MMC in the two studies. Second, the incidence and severity of corneal haze as a LASEK complication is reported to peak at 1 month to 3 months and resolve to a low grade after 6 months [9] [15] [17]. If we followed up the stability for a longer time, the rate of losing two or more lines of BSCVA might become lower, which is why our report has a weak point. The third possibility is that only 561 of the original 821 patients enrolled in our study attended follow-up sessions, necessitating exclusion of the other 260 patients. If we presume that these 260 patients were satisfied with the outcome of their LASEK operation, which is a likely explanation for their failure to attend follow-up sessions, this would have skewed the results toward an increased incidence rate of losing two or more lines of BSCVA.

The causes of loss of BSCVA after refractive surgery are thought to be loss of corneal clarity secondary to haze or scarring, irregular astigmatism secondary to eccentric ablation, a central island or corneal ectasia, flaprelated complications and epithelial in-growth. Because the LASEK procedure in particular results in cells losing their integrity and capacity to adhere and recover, corneal haze does influence visual outcomes and is a main reason for loss of BSCVA. In our study, corneal haze should also be a main reason for loss of BSCVA, because corneal haze contributed to 24 of 47 (51.1\%) and 19 of 29 (65.5\%) eyes of those that lost two or more lines of BSCVA at 3 months and 6 months postoperatively, respectively. Thus, some risk is inescapable with a LASEK procedure [8] [15] and, therefore, use of MMC might lead to widespread reduction in the incidence of this complication. Nevertheless, when applied to the cornea, MMC could potentially damage corneal epithelial [18] [19], stromal cells [20] [21], and endothelial cells [22] [23], so the possible toxicity of this drug remains a subject of intense debate [11].

To determine risk factors for corneal haze, the correlation between the grade of the corneal haze and other clinical parameters, which are degrees of preoperative myopia, pre- and postoperative corneal thickness, and the diameter and depth of ablation, should be evaluated. It is very difficult to evaluate the grade of corneal haze in each enrolled patient retrospectively. Instead, because the degree of preoperative myopia may be one risk factor for postoperative haze, we studied the correlation between loss of two or more lines of BSCVA and the degree of preoperative myopia. As shown in Figure 1, the decimal VA converted from the mean logMAR of the postoperative BSCVA was between 1.2 and 1.5 in all myopia groups; therefore, LASEK seems to be safe in terms of obtaining mean BSCVAs of $>1.2$ with all preoperative myopia groups. However, when we compared the incidence rate of losing two or more lines of BSCVA, we found the high or ultra-high myopia groups had a signifi- 
cantly higher incidence rate than the low or mild myopic groups at 3 months or 6 months postoperatively. Our results suggested that LASEK is less safe in terms of minimal loss of VA in high to ultra-high myopia groups than in groups with less pronounced myopia. LASEK seems to have increased risk when the preoperative SE is more than 6.125 D. LASEK is a viable option for many surgeons to avoid flap-related complications, including dissection of corneal nerves [24]-[26] and retinal tear production caused by the suction ring of the microkeratome. Consequently, we consider LASEK as more safely performed in patients with less than $6.00 \mathrm{D}$ of preoperative SE.

\section{Acknowledgements}

We thank Professor Masaaki Goto for his statistical support and Ms. Keiko Miyazaki, Mr. Yohei Yoshimoto, Mr. Kohei Kumagai, Mr. Takeshi Tominaga and Mr. Ryo Ikemi for clinical support. Nurses Ms. Setsuko Kamachi, Ms. Aki Suezaki, Ms. Yumi Nozaki, Ms. Masami Yamamoto, and Ms. Miyuki Ito provided excellent assistance during the LASEK operations, for which we thank them.

\section{References}

[1] Azar, D.T., Ang, R.T., Lee, J.B., Kato, T., Chen, C.C., Jain, S., Gabison, E. and Abad, J.C. (2001) Laser Subepithelialkeratomileusis: Electron Microscopy and Visual Outcomes of Flap Photorefractive Keratectomy. Current Opinion in Ophthalmology, 12, 323-328. http://dx.doi.org/10.1097/00055735-200108000-00014

[2] Taneri, S., Feit, R. and Azar, D.T. (2004) Safety, Efficacy, and Stability Indices of LASEK Correction in Moderate Myopia and Astigmatism. Journal of Cataract Refractive Surgery, 30, 2130-2137.

http://dx.doi.org/10.1016/j.jcrs.2004.02.070

[3] Claringbold 2nd, T.V. (2002) Laser-Assisted Subepithelial Keratectomy for the Correction of Myopia. Journal of Cataract Refractive Surgery, 28, 18-22. http://dx.doi.org/10.1016/S0886-3350(01)01234-2

[4] Rouweyha, R.M., Chuang, A.Z., Mitra, S., Phillips, C.B. and Yee, R.W. (2002) Laser Epithelial Keratomileusis for Myopia with the Autonomous Laser. Journal of Refractive Surgery, 18, 217-224.

[5] Autrata, R. and Rehurek, J. (2004) Laser-Assisted Subepithelial Keratectomy and Photorefractive Keratectomy versus Conventional Treatment of Myopic Anisometropic Amblyopia in Children. Journal of Cataract Refractive Surgery, 30, 74-84. http://dx.doi.org/10.1016/S0886-3350(03)00417-6

[6] Kim, J.K., Kim, S.S., Lee, H.K., Lee, I.S., Seong, G.J., Kim, E.K. and Han, S.H. (2004) Laser in Situ Keratomileusis versus Laser-Assisted Subepithelial Keratectomy for the Correction of High Myopia. Journal of Cataract Refractive Surgery, 30, 1405-1411. http://dx.doi.org/10.1016/j.jcrs.2003.12.053

[7] Taneri, S., Zieske, J.D. and Azar, D.T. (2004) Evolution, Techniques, Clinical Outcomes, and Pathophysiology of LASEK: Review of the Literature. Survey of Ophthalmology, 49, 576-602. http://dx.doi.org/10.1016/j.survophthal.2004.08.003

[8] Taneri, S., Weisberg, M. and Azar, D. (2011) Surface Ablation Techniques. Journal of Cataract Refractive Surgery, 37, 392-408. http://dx.doi.org/10.1016/j.jcrs.2010.11.013

[9] Feit, R., Taneri, S., Azar, D.T., Chen, C.C. and Ang, R.T. (2003) LASEK Results. Ophthalmology Clinics of North America, 16, 127-135. http://dx.doi.org/10.1016/S0896-1549(02)00063-9

[10] Vinciguerra, P. and Camesasca, F. (2002) Butterfly Laser Epithelial Keratomileusis for Myopia. Journal of Refractive Surgery, 18 [Suppl], 371-373.

[11] Teus, M.A., de Benito-Llopis, L. and Alió, J.L. (2009) Mitomycin C in Corneal Refractive Surgery. Survey of Ophthalmology, 54, 487-502. http://dx.doi.org/10.1016/j.survophthal.2009.04.002

[12] Chen, S.H., Feng, Y.F., Stojanovic, A. and Wang, Q.M. (2011) Meta-Analysis of Clinical Outcomes Comparing Surface Ablation for Correction of Myopia with and without $0.02 \%$ Mitomycin C. Journal of Refractive Surgery, 27, 530-541. http://dx.doi.org/10.3928/1081597X-20110112-02

[13] Ghirlando, A., Gambato, C. and Midena, E. (2007) LASEK and Photorefractive Keratectomy for Myopia: Clinical and Confocal Microscopy Comparison. Journal of Refractive Surgery, 23, 694-702.

[14] Pirouzian, A., Thornton, J.A. and Ngo, S. (2004) A Randomized Prospective Clinical Trial Comparing Laser Subepithelial Keratomileusis and Photorefractive Keratectomy. Archives of Ophthalmology, 122, 11-16. http://dx.doi.org/10.1001/archopht.122.1.11

[15] Zhao, L.Q., Wei, R.L., Cheng, J.W., Li, Y., Cai, J.P. and Ma, X.Y. (2010) Meta-Analysis: Clinical Outcomes of Laser-Assisted Subepithelial Keratectomy and Photorefractive Keratectomy in Myopia. Ophthalmology, 117, 1912-1922. http://dx.doi.org/10.1016/j.ophtha.2010.02.004 
[16] de Benito-Llopis, L., Teus, M.A., Gil-Cazorla, R. and Drake, P. (2009) Comparison between Femtosecond Laser-Assisted Sub-Bowman Keratomileusis vs Laser Subepithelial Keratectomy to Correct Myopia. American Journal of Ophthalmology, 148, 830-836. http://dx.doi.org/10.1016/j.ajo.2009.07.008

[17] Moller-Pedersen, T., Cavanagh, H.D., Petroll, W.M. and Jester, J.V. (2000) Stromal Wound Healing Explains Refractive Instability and Haze Development after Photorefractive Keratectomy: A 1-Year Confocal Microscopic Study. Ophthalmology, 107, 1235-1245. http://dx.doi.org/10.1016/S0161-6420(00)00142-1

[18] Kymionis, G.D., Tsiklis, N.S., Ginis, H., Diakonis, V.F. and Pallikaris, I. (2006) Dry Eye after Photorefractive Keratectomy with Adjuvant Mitomycin C. Journal of Refractive Surgery, 22, 511-513.

[19] Lee, D.H., Chung, H.S., Jeon, Y., Boo, S.D., Yoon, Y.D. and Kim, J.G. (2005) Photorefractive Keratectomy with Intraoperative Mitomycin-C Application. Journal of Cataract \& Refractive Surgery, 31, 2293-2298. http://dx.doi.org/10.1016/j.jcrs.2005.05.027

[20] Netto, M.V., Mohan, R.R., Sinha, S., Sharma, A., Gupta, P.C. and Wilson, S.E. (2006) Effect of Prophylactic and Therapeutic Mitomycin C on Corneal Apoptosis, Cellular Proliferation, Haze, and Long-Term Keratocyte Density in Rabbits. Journal of Refractive Surgery, 22, 562-574.

[21] Rajan, M.S., O’Brart, D.P.S., Patmore, A. and Marshall, J. (2006) Cellular Effects of Mitomycin-C on Human Corneas after Photorefractive Keratectomy. Journal of Cataract \& Refractive Surgery, 32, 1741-1747. http://dx.doi.org/10.1016/j.jcrs.2006.05.014

[22] Morales, A.J., Zadok, D., Mora-Retana, R., Martínez-Gama, E., Robledo, N.E. and Chayet, A.S. (2006) Intraoperative Mitomycin and Corneal Endothelium after Photorefractive Keratectomy. American Journal of Ophthalmology, 142, 400-404. http://dx.doi.org/10.1016/j.ajo.2006.04.029

[23] Nassiri, N., Farahangiz, S., Rahnavardi, M., Rahmani, L. and Nassiri, N. (2008) Corneal Endothelial Cell Injury Induced by Mitomycin-C in Photorefractive Keratectomy: Nonrandomized Controlled Trial. Journal of Cataract \& Refractive Surgery, 34, 902-908. http://dx.doi.org/10.1016/j.jcrs.2008.03.007

[24] Matsui, H., Kumano, Y., Zushi, I., Yamada, T., Matsui, T. and Nishida, T. (2001) Corneal Sensation after Correction of Myopia by Photorefractive Keratectomy and Laser in Situ Keratomileusis. Journal of Cataract \& Refractive Surgery, 27, 370-373. http://dx.doi.org/10.1016/S0886-3350(00)00756-2

[25] Kumano, Y., Matsui, H., Zushi, I., Mawatari, A., Matsui, T., Nishida, T. and Miyazaki, M. (2003) Recovery of Corneal Sensation after Myopic Correction by Laser in Situ Keratomileusis with a Nasal or Superior Hinge. Journal of Cataract \& Refractive Surgery, 29, 757-761. http://dx.doi.org/10.1016/S0886-3350(02)01840-0

[26] Lee, S.J., Kim, J.K., Seo, K.Y., Kim, E.K. and Lee, H.K. (2006) Comparison of Corneal Nerve Regeneration and Sensitivity between LASIK and Laser Epithelial Keratomileusis (LASEK). American Journal of Ophthalmology, 141, 1009-1015. http://dx.doi.org/10.1016/j.ajo.2006.01.048 
Scientific Research Publishing (SCIRP) is one of the largest Open Access journal publishers. It is currently publishing more than 200 open access, online, peer-reviewed journals covering a wide range of academic disciplines. SCIRP serves the worldwide academic communities and contributes to the progress and application of science with its publication.

Other selected journals from SCIRP are listed as below. Submit your manuscript to us via either submit@scirp.org or Online Submission Portal.
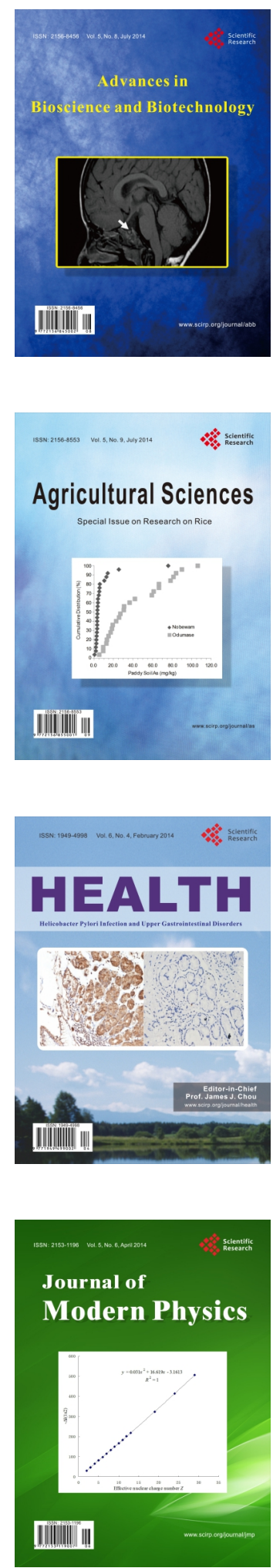
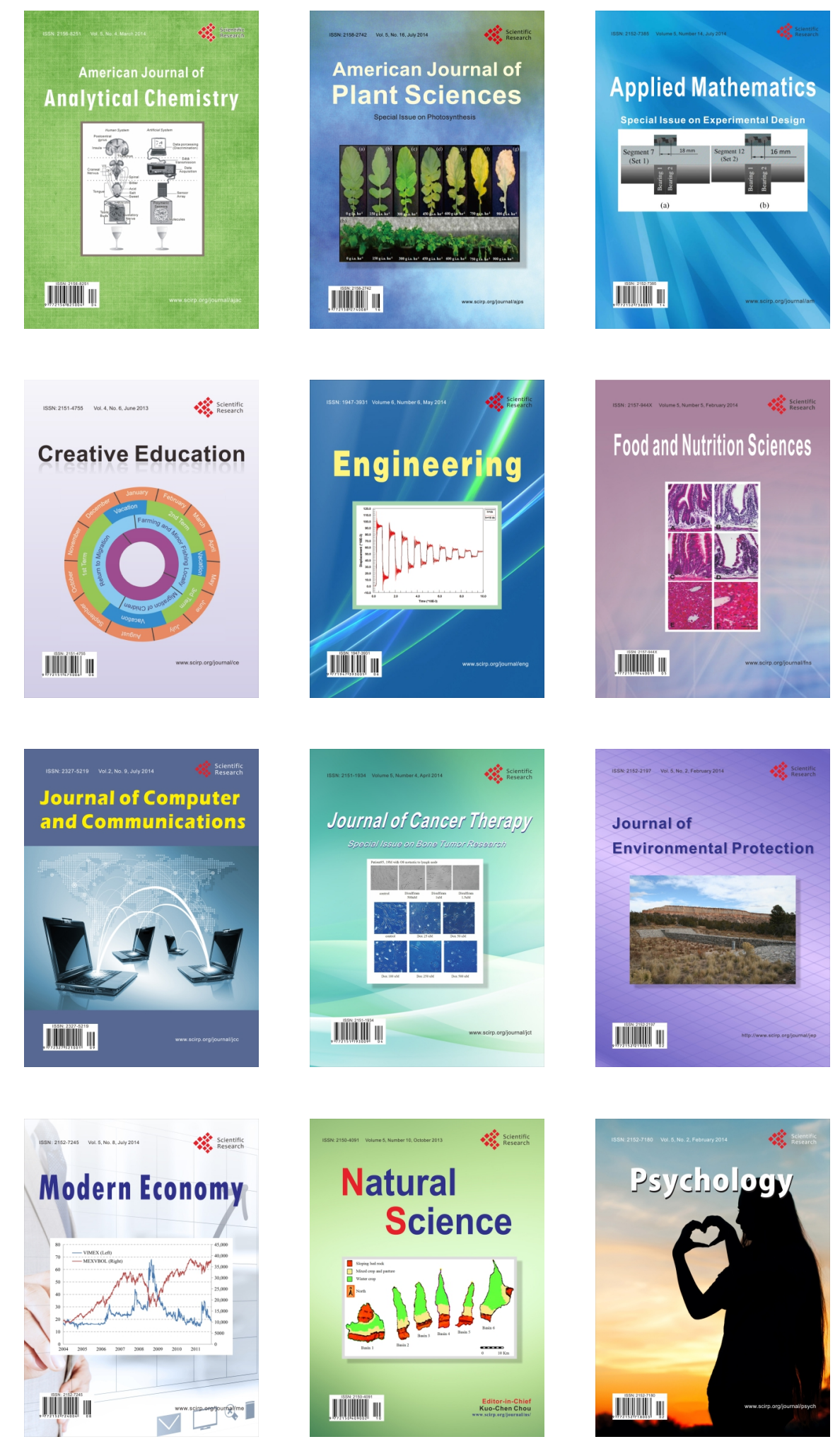